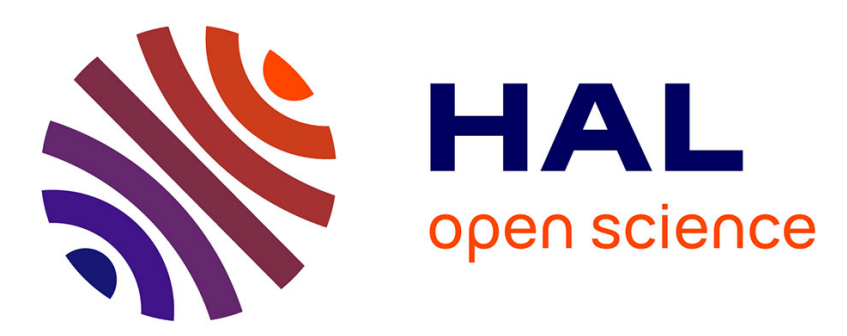

\title{
Maintenance on the Railway Network: Disruptions and re-scheduling
}

Samuel Deleplanque

\section{To cite this version:}

Samuel Deleplanque. Maintenance on the Railway Network: Disruptions and re-scheduling. Electronic Notes in Discrete Mathematics, 2018, 69, pp109-116. hal-01871120

\section{HAL Id: hal-01871120 \\ https://hal.science/hal-01871120}

Submitted on 10 Jun 2021

HAL is a multi-disciplinary open access archive for the deposit and dissemination of scientific research documents, whether they are published or not. The documents may come from teaching and research institutions in France or abroad, or from public or private research centers.
L'archive ouverte pluridisciplinaire HAL, est destinée au dépôt et à la diffusion de documents scientifiques de niveau recherche, publiés ou non, émanant des établissements d'enseignement et de recherche français ou étrangers, des laboratoires publics ou privés. 


\title{
Maintenance on the Railway Network: Disruptions and re-scheduling
}

\author{
Samuel Deleplanque ${ }^{\mathrm{a}, \mathrm{b}, \mathrm{c}}$ \\ a IFSTTAR, COSYS, ESTAS \\ Lille Nord de France University \\ rue Elisée Reclus 20, 59666 Villeneuve d'Ascq \\ Lille, France \\ samuel.deleplanque@ifsttar.fr \\ b Département d'Informatique \\ Université Libre de Bruxelles \\ Brussels, Belgium \\ c INOCS, INRIA Lille Nord-Europe, France
}

\begin{abstract}
Many studies had been made on optimization problems related to the maintenance operation works in the railway world. In this paper, we focus on lesser-known type of these problems. Here, the maintenance is already scheduled and the infrastructure manager can only change the train timetables and the routing. This problem is not well treated in the literature. In this work, we define the problem and we present a model. Since several days are available to solve the problem, the level of abstraction of the model and the size of the considered instances let us use an exact method. We propose a Mixed Integer Linear Program minimizing the primary delays. Results on real world instances are reported.
\end{abstract}

Keywords: Scheduling, Railway, Maintenance Works, Mixed Integer Programming. 


\section{Introduction}

In European railway systems, some Infrastructure Managers (IM) need a rescheduling tool which automatically updates the original timetables and routings, when some maintenance operations must be made on the network. Some of these works can be scheduled a long time in advance, sometimes while the timetables are made, and some other works can be scheduled while the rail is not used (e.g. by night). However, the maintenance operations considered in this paper have to be done at a specific time. The IM can only make a change on the timetables for the trains which normally use the same track and during the same time window of the works.

This specific re-scheduling process is often made by hand, and it is not usually well optimized when the traffic is dense. In this work, we propose an optimization model for modifying the timetables in order to minimize the delays while the work maintenance can be completed on time.

The main difficulties are the number of constraints, the size of the network, the quantity of trains and many other features related to the considered railway system. These difficulties imply that some choices have to be made. For example, one can reduce the space and the time window where and when trains can be rescheduled. However, the maintenance works considered in this paper are planned 3 weeks in advance. In this work, the level of abstraction of the model and the time available for solving the instances considered allow us to implement an exact method.

Our optimization model is based on a Mixed Integer Linear Program (MILP). It can take into account several maintenance operations for a given time window and for a given area. The direction of the optimization in the experiments is the minimization of the arrival delays.

The literature on the delay management had been particularly prolific. Minimizing primary or secondary delays is an important topic in the Operation Research literature applied on railway problems. In [1] and [2], the authors propose a way to decide which connecting trains should wait for a delayed train. They consider the propagation of the delays in order to know if it is relevant for these trains to wait knowing that they will arrive with another delay at the next station where other passengers want to transfer. These recent works include several aspects of the real world which are not usually considered in the literature. For example, they consider different waiting times according to the number of people and the moment of the day. If a large flow of people is transferred to a train, the waiting time would be different comparing with a small flow of people. And, for the same number of people, the flows 
could be more or less fast at different moments of the days.

In [2], the optimization model takes the point of view of the passengers. Here, when a transfer is planned, the system takes into account the possibility that a passenger might take another train if the primary delay (of his first train) makes the second train schedule not optimal for the passenger since taking another train can be a better solution at the station where the transfer will be made.

This work is focusing mainly on primary delays since there are not any connections considered in the instances. Minimizing delays had been studied in several contexts included when the maintenance works must be done.

In [3], the authors define two separated sets: the complete blockades, where all the tracks are blocked and the partial blockades, where one or few tracks are not available. This paper proposes a method to handle the disturbances for the second case. The main contribution is a static optimization model based on a model of [4] and adapted for our specific problem.

This paper is structured as follows. In section 2, we first present the train maintenance problem considered in this work. In section 3, the optimization model is formally defined by a Mixed Integer Program. Finally, in section 4, we describe the experimental setup and discuss the results.

\section{Problem definition}

This work considers a problem of re-scheduling in a railway system. Some maintenance operations had been planned during a specific period on some part of a track. At that period, the trains which are usually running on that track will have to be rerouted and/or postponed.

The model presented in this paper takes into account several parallel tracks with some switches allowing the trains to change from one track to another. As in Figure 1, the other switches are not taken into account.

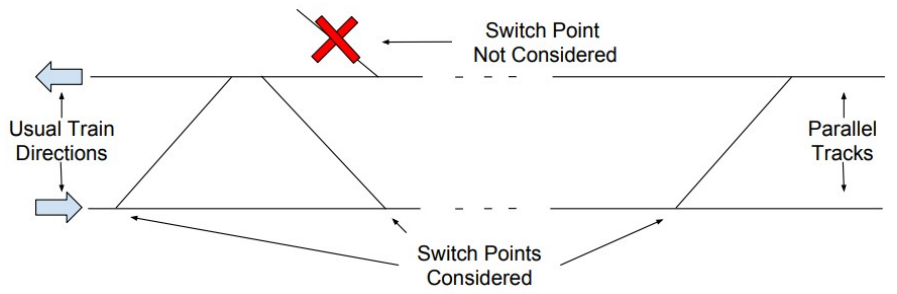

Fig. 1. Switch points considered in the model 


\subsection{Definitions}

We need to define the notions of segment and event which are two main concepts used in the problem formulation:

- A segment is a specific portion of a railway network. A segment gathers all the parallel tracks between two points A and B. In a given segment, it is impossible to go from one track to another: the trains can only change their routes at one end of the segment. Such a segment is composed of one or several tracks. There are often 2 tracks in a real railway network. In this case, we have one way for each direction. Also, some segments represent only one point $(\mathrm{A}=\mathrm{B})$ if they are related to the idle trains.

- An event is the process of a train going from a point $\mathrm{A}$ to a point B by a segment. One event is linked with exactly one segment and only one train. The related track is chosen by the IM and it is modifiable in our problem. An event is also defined with a departure time and an arrival time. When the segment of this event corresponds to a single point, it represents the waiting time of the train at this point.

\subsection{Problem data}

The following model is an adaptation of the one proposed in [4]. Some constraints have been removed. For instance, we don't check if a train can fit in the track section modeled by a event. Conversely, some other constraints had been added mainly to integrate the works.

We define first the parameters obtained from the input. We note $R, E$, $W$ and $S$ the sets of train routes, events, works and segments, respectively. In the same order, these sets are indexed by $r, e, w$ and $s . S E_{s}, s \in S$, is a subset of $E$ the set of events. This is the ordered list of events on all the tracks of the given segment $s . R E_{r}, r \in R$, is another subset of $E$. It corresponds to the ordered list of events on the train route $r$. The last event for a given $r$ in $R E_{r}$ is noted $n_{r}$. The orders of $S E_{s}, s \in S$, and $R E_{r}, r \in R$, follow the original timetable in chronological order.

We denote by $T_{s}$ the set of tracks $t, t=1 . .\left|T_{s}\right|$, for a given segment $s, s \in S$. For any segment $s, s \in S$, the model considers two sets of positive constants called separation time. $\Delta_{s}^{F}$ is the minimum time separating two consecutive trains following each other and, $\Delta_{s}^{M}$ is the minimum time separating two trains in opposite direction.

In the original timetable, an event $e, e \in E$, starts initially at time $\tau_{e}^{\text {begin }}$ and finishes at time $\tau_{e}^{e n d}$. According to the type of an event $e, e \in E$, the 
parameter $d_{e}$ describes the minimum running time or a minimum waiting time. The first time is related to an event $e, e \in E$, with a train running from one point to another (this situation is modeled with the equality $i_{e}=0$ ). The second time corresponds to the stop duration at a point $\left(i_{e}=1\right)$.

The constant $o_{e}$ indicates the direction of the train for the event $e, e \in$ $E$. For instance, two consecutive events $e, e \in E$, and $e^{\prime}, e^{\prime} \in E$, with the related train route $r, r \in R$, and $r^{\prime}, r^{\prime} \in R$, following each other, imply the equality $o_{e}=o_{e^{\prime}}$. Otherwise, if the trains are moving in opposite direction, we have $o_{e} \neq o_{e^{\prime}}$. A work maintenance $w_{t}, w_{t} \in W$, has a time window noted $\left[w_{t}^{\text {begin }} ; w_{t}^{\text {end }}\right]$. We consider that a given track $t, t \in T_{s}$, on a segment $s \in S$, is limited to only one work.

We now define the 7 sets of variables. The 3 first sets are containing continuous variables:

- $x_{e}^{\text {begin }}$ is the time when the event $e, e \in E$, starts;

- $x_{e}^{e n d}$ is the time when the event $e, e \in E$, ends;

- $z_{e}$ is the delay of the event $e, e \in E$.

The 4 other sets contain binary variables:

- $q_{e t}$ is equal to 1 if the event $e, e \in S E_{s}, s \in S$, is planned on track $t, t \in$ $T_{s}, s \in S, 0$ otherwise;

- $\alpha_{e}^{w_{t}}$ is equal to 1 if the event $e, e \in E$, will be scheduled before the fixed maintenance work $w, w \in W, 0$ otherwise;

- $\gamma_{e e ̂}$ is equal to 1 if, as in the original timetable, $e$ is located before $\hat{e}$ on the events ordered list $S E_{s}, S E_{s} \in E, s \in S, 0$ otherwise;

- $\lambda_{e \hat{e}}$ is equal to 1 if, on the contrary to the original timetable, $\hat{e}$ is located before $e$ on the events ordered list $S E_{s}, S E_{s} \in E, s \in S, 0$ otherwise.

\section{Optimization model}

We present here the optimization model. First we detail the constraints and second we define the objective function.

\subsection{Constraints}

For a given train, a series of events is controlled by Constraints (1) which force an event to start once the previous one is finished. Constraints (2) give a lower bound on the event duration. Here, a train can speed up but can't be 
faster than the minimum time $d_{e}$ of the event.

$$
\begin{gathered}
x_{e}^{e n d}=x_{e+1}^{\text {begin }}, \quad e \in R E_{r}, r \in R: e=1 . . n_{r}-1, \\
x_{e}^{\text {end }} \geq x_{e}^{\text {begin }}+d_{e}, \quad e \in E,
\end{gathered}
$$

In Constraints (3), an event $e$ cannot be scheduled before it had been already scheduled in the timetable by $\tau_{e}^{\text {begin }}$ while Constraints (4) just give the delay value of an event $e$ to $z_{e}$.

$$
\begin{gathered}
x_{e}^{\text {begin }} \geq \tau_{e}^{\text {begin }}, \quad e \in E: i_{e}=1, \\
x_{e}^{\text {end }}-\tau_{e}^{\text {end }} \leq z_{e}, \quad e \in E,
\end{gathered}
$$

For each event of each segment, Constraints (5) force the usage of one and only one track.

$$
\sum_{t \in T_{s}} q_{e t}=1, \quad e \in S E_{s}, s \in S
$$

Constraints (6) and (7) force the events planned during the work maintenance to be rescheduled. If such an event $e$ is still planned on the same track of the work $w$, Constraints (6) will force $e$ to start after the end of $w$ or Constraints (7) will force $e$ to finish before the beginning of $w$. The big-M will activate one or the other constraint.

$$
\begin{gathered}
x_{e}^{\text {begin }} \geq w_{t}^{e n d} q_{e t}-M \alpha_{e}^{w_{t}}, \quad e \in S E_{s}, t \in T_{s}, s \in S, \\
x_{e}^{e n d} \leq w_{t}^{\text {begin }} q_{e t}+M\left(1-\alpha_{e}^{w_{t}}\right), \quad e \in S E_{s}, t \in T_{s}, s \in S,
\end{gathered}
$$

Constraints (8) and (9) are made to activate the Constraints $(10,11,12$, 13) by giving values to $\lambda_{e \hat{e}}$ and $\gamma_{e \hat{e}}$ according to the order of events in case they are scheduled on the same tracks.

$$
\begin{gathered}
q_{e t}+q_{\hat{e} t}-1 \leq \lambda_{e \hat{e}}+\gamma_{e \hat{e}}, \quad e, \hat{e} \in S E_{s}, t \in T_{s}, s \in S: e<\hat{e}, \\
\lambda_{e \hat{e}}+\gamma_{e \hat{e}} \leq 1, \quad e, \hat{e} \in S E_{s}, s \in S: e<\hat{e}
\end{gathered}
$$

The 4 sets of Constraints $(10,11,12,13)$ activate the separation times on two consecutive trains for a given track. If the trains are running in opposite direction $\left(o_{e} \neq o_{\hat{e}}\right)$, the Constraints $(10,12)$ apply a separation time $\Delta_{s}^{M}$ for the segment $s$. Otherwise, if the trains are running in the same direction $\left(o_{e}=o_{\hat{e}}\right)$, the Constraints $(11,13)$ apply a shorter separation time $\Delta_{s}^{F}$ of the same segment $s$. 


$$
\begin{aligned}
& x_{\hat{e}}^{\text {begin }}-x_{e}^{e n d} \geq \Delta_{s}^{M} \gamma_{e \hat{e}}-M\left(1-\gamma_{e \hat{e}}\right), e<\hat{e} \in S E_{s}, s \in S, o_{e} \neq o_{\hat{e}}, \\
& x_{\hat{e}}^{\text {begin }}-x_{e}^{e n d} \geq \Delta_{s}^{F} \gamma_{e \hat{e}}-M\left(1-\gamma_{e \hat{e}}\right), e<\hat{e} \in S E_{s}, s \in S, o_{e}=o_{\hat{e}}, \\
& x_{e}^{\text {begin }}-x_{\hat{e}}^{e n d} \geq \Delta_{s}^{M} \lambda_{e \hat{e}}-M\left(1-\lambda_{e \hat{e}}\right), e<\hat{e} \in S E_{s}, s \in S, o_{e} \neq o_{\hat{e}}, \\
& x_{e}^{\text {begin }}-x_{\hat{e}}^{e n d} \geq \Delta_{s}^{F} \lambda_{e \hat{e}}-M\left(1-\lambda_{e \hat{e}}\right), e<\hat{e} \in S E_{s}, s \in S, o_{e}=o_{\hat{e}},
\end{aligned}
$$

The binary and continuous variables are finally defined by Constraints (14, $15,16)$.

$$
\begin{gathered}
x_{e}^{\text {begin }}, x_{e}^{e n d}, z_{e} \geq 0, \quad e \in E, \\
\gamma_{e \hat{e}}, \lambda_{e \hat{e}}, \in\{0,1\}, \quad e, \hat{e} \in S E_{s}, s \in S: e<\hat{e}, \\
q_{e t}, \alpha_{e}^{w_{t}} \in\{0,1\}, \quad e \in S E_{s}, t \in T_{s}, s \in S,
\end{gathered}
$$

\subsection{Objective function}

We define a simple objective function which minimizes the final delays of the trains. We note $z_{n_{r}}$ the delay variable of the last event considered in the instance for the train $r$. The objective function is the following:

$$
\min \sum_{r \in R} z_{n_{r}}
$$

\section{Experiments}

The instances are based on real data from the Belgian network. The instances are randomly chosen in terms of time and space. However, the size of these instances are based on what the IM needs to solve in general. We consider here the typical case with two parallel tracks and with 4 or 5 switch points. Periods from 10 hours to 18 hours are considered. The instances had been solved by CPLEX 12.6 running on a $3 \mathrm{Ghz}$ Intel i7 CPU.

Table 1 shows the 2 sets of 9 instances. CPU times in seconds and the number of nodes needed by CPLEX are given for each time windows (TW) in hours.

These instances are tackled within 10 seconds including the ones covering a full day of exploitation. The efficiency of the optimization model seems to be sufficient since these maintenance operations are known weeks in advance. 


\begin{tabular}{r|rr|rr} 
& \multicolumn{2}{|c|}{ 4 switch points } & \multicolumn{2}{c}{5 switch points } \\
\hline TW (h) & CPU(s) & Nodes & CPU(s) & Nodes \\
\hline 10 & 0.91 & 1 & 0.90 & 79 \\
11 & 1.15 & 1 & 1.36 & 279 \\
12 & 1.26 & 1 & 1.95 & 87 \\
13 & 0.95 & 1 & 7.60 & 1367 \\
14 & 0.89 & 1 & 1.64 & 151 \\
15 & 1.17 & 82 & 8.50 & 959 \\
16 & 2.73 & 65 & 1.54 & 73 \\
17 & 2.05 & 49 & 5.84 & 498 \\
18 & 3.53 & 138 & 4.73 & 224
\end{tabular}

Computational Results on the instances with 4 and 5 switch points

\section{Acknowledgment}

This project is studied at the Universite Libre de Bruxelles in collaboration with INFRABEL, the Belgian railway infrastructure manager. It is financed by the COFUND program of the European Union (FP7 - Marie Curie Actions) with the Beware Academia Fellowship.

\section{References}

[1] T. Dollevoet. Delay management and dispatching in railways. Number EPS2013-272-LIS. 2013.

[2] T. Dollevoet, D. Huisman, M. Schmidt, and A. Schöbel. Delay management with rerouting of passengers. Transportation Science, 46(1):74-89, 2012.

[3] I. Louwerse and D. Huisman. Adjusting a railway timetable in case of partial or complete blockades. European Journal of Operational Research, 235(3):583 $593,2014$.

[4] J. Törnquist and J. A Persson. N-tracked railway traffic re-scheduling during disturbances. Transportation Research Part B: Methodological, 41(3):342-362, 2007. 